\title{
On fusion for multispectral iris recognition
}

Conference or Workshop Item

Accepted Version

Wild, P., Radu, P. and Ferryman, J. (2015) On fusion for multispectral iris recognition. In: 8th IAPR International Conference on Biometrics (ICB2015), 19-22 May, 2015, Phuket, Thailand, pp. 31-73. Available at http://centaur.reading.ac.uk/48396/

It is advisable to refer to the publisher's version if you intend to cite from the work. See Guidance on citing.

Published version at: http://ieeexplore.ieee.org/xpls/abs_all.jsp?arnumber $=7139072$

All outputs in CentAUR are protected by Intellectual Property Rights law, including copyright law. Copyright and IPR is retained by the creators or other copyright holders. Terms and conditions for use of this material are defined in the End User Agreement.

www.reading.ac.uk/centaur 


\section{CentAUR}

Central Archive at the University of Reading

Reading's research outputs online 


\title{
On Fusion for Multispectral Iris Recognition
}

\author{
Peter Wild, Petru Radu, James Ferryman \\ Computational Vision Group, School of Systems Engineering, University of Reading, UK \\ \{p.wild, p.radu, j.m.ferryman\}@reading.ac.uk
}

\begin{abstract}
Multispectral iris recognition uses information from multiple bands of the electromagnetic spectrum to better represent certain physiological characteristics of the iris texture and enhance obtained recognition accuracy. This paper addresses the questions of single versus crossspectral performance and compares score-level fusion accuracy for different feature types, combining different wavelengths to overcome limitations in less constrained recording environments. Further it is investigated whether Doddington's "goats" (users who are particularly difficult to recognize) in one spectrum also extend to other spectra. Focusing on the question of feature stability at different wavelengths, this work uses manual ground truth segmentation, avoiding bias by segmentation impact. Experiments on the public UTIRIS multispectral iris dataset using 4 feature extraction techniques reveal a significant enhancement when combining NIR + Red for 2-channel and NIR + Red + Blue for 3-channel fusion, across different feature types. Selective feature-level fusion is investigated and shown to improve overall and especially cross-spectral performance without increasing the overall length of the iris code.
\end{abstract}

\section{Introduction}

Traditional visual biometrics uses either monochrome or 3-channel RGB colour images for sensing biometric traits. Emerging multispectral techniques exploit multiple bands of the electro-magnetic spectrum in the recognition process, aiming for enhanced accuracy when information is combined [15], or predicting performance in unknown channels [21] for easier or cheaper recording of biometric signals. Especially in iris recognition due to the narrow depth of field in optical setups and illumination problem in visible range (strong reflections) any means to enhance the entropy of biometric source signals is highly appreciated. Further, benefits of multispectral imaging comprise the efficient encoding of complementary information for counterspoofing [4], combination of the iris biometric with conjunctival vasculature, and potential cross-spectral applications with quantum photography in the future [12].
Most of the commercial implementations of iris recognition systems use iris images acquired in the near infrared (NIR) spectrum in the $700 \mathrm{~nm}$ to $900 \mathrm{~nm}$ range. With the recent challenge evaluations on noisy iris images (NICE), considerable focus of research groups has also been put on the visible wavelength (VW) spectrum at $390 \mathrm{~nm}$ to $700 \mathrm{~nm}$, especially for the purpose of robust iris segmentation. This task turns out to be particularly challenging for dark (highly pigmented) iris images, due to very low contrast between pupil and sclera, and certainly due to the presence of strong reflections which can hardly be controlled in this band. The former reason, more specifically the presence of Eumelanin (brown-black melanin) and Pheomelanin (yellow-red melanin) in dark eyes, make recognition in the visible spectrum particularly difficult for this type of eyes. On the other hand, for less pigmented iris images, visible range images show incredibly rich texture, see Fig. 1 comparing a hazel and brown iris in both spectra.

While iris multispectral fusion has attracted researchers to look into far NIR (greater than 900nm) bands [19] and NIR+VW bands [15], it is especially the combination of NIR images with images captured in VW (in particular the red channel being highly discriminative [1]) which is of particular interest due to cheap available sensors and illuminators for this type of imagery. As a contribution to existing multispectral evaluations, this paper presents an efficient feature-level fusion using random selected bits [17] and thoroughly addresses the following questions: (1) How is intra and inter-channel performance affected for different types of features? (2) Are difficult subjects in one spectrum also difficult in another? (3) Which features and combinations thereof are most discriminatory for different wavelengths?

Section 2 gives a short survey on multispectral iris recognition, highlighting the most relevant related works. Employed baseline algorithms, the multispectral database and tested fusion approaches are introduced in Section 3. Section 4 analyses experimental results with regards to questions outlined above. Finally, the conclusion and wrap-up of main insights with regards to our multispectral fusion experiments is outlined in Section 5. 


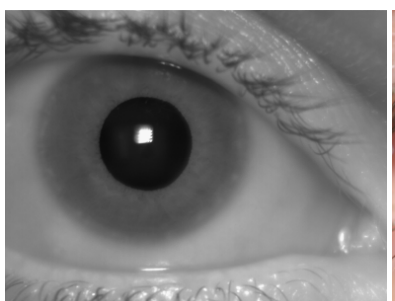

(a) hazel eye \#28R_3 in NIR

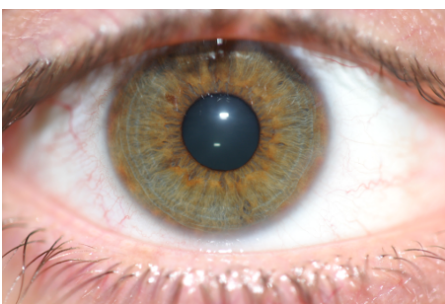

(b) hazel eye \#28R_2 in VW

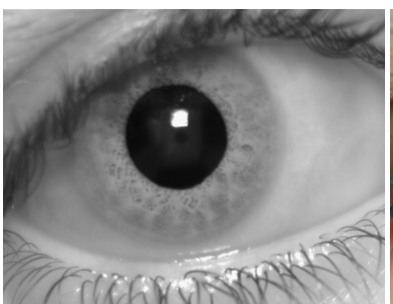

(c) brown eye \#27R_1 in NIR

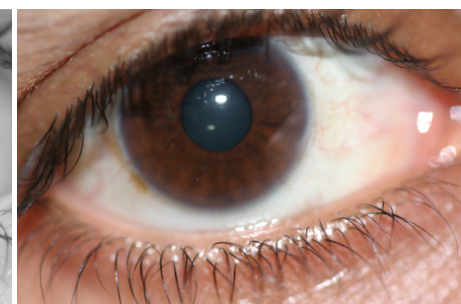

(d) brown eye \#27R_1 in VW

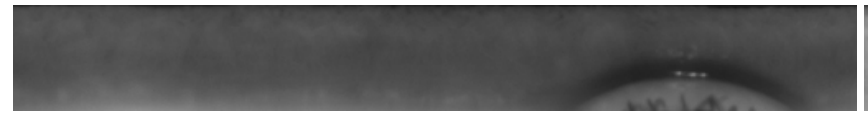

(e) normalised texture of hazel eye \#28R_3 in NIR

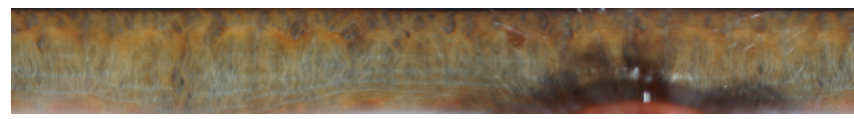

(g) normalised texture of hazel eye \#28R_2 in VW

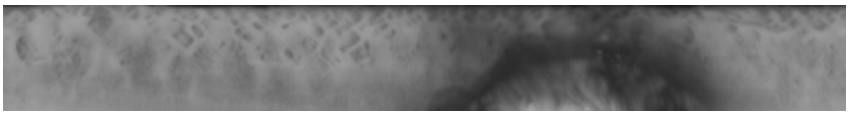

(f) normalised texture of brown eye \#27R_1 in NIR

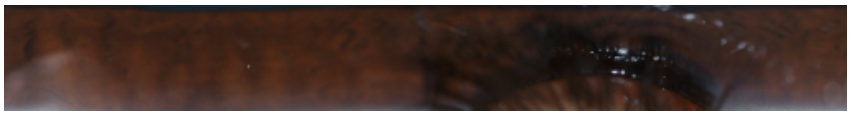

(h) normalised texture of brown eye \#27R_1 in VW

Figure 1: Iris texture in VW can reveal even richer texture (high-frequency patterns) than in NIR for light irises (e.g., hazel eyes), whereas for heavily pigmented dark (brown) irises clear patterns are revealed in NIR (samples from UTIRIS database).

\section{Related Work}

Joint NIR and VW iris acquisition is patented by Burge and Monaco [3], combining 460nm, 555nm, 670nm and $800 \mathrm{~nm}$ unwrapped bands and clustering the image at pixellevel. The patent lists claims for methods to compare the enhanced iris image against the VW or NIR image only, as well as transformations to generate a NIR representation from the VW image, and vice-versa, and the generation of $3 \mathrm{D}$ depth maps generated from stereo images. Their singlelens spatially-registered 4-camera ( 2 x NIR, 2x VW) system is further described in [2], introducing the notion of Multispectral Enhanced IrisCode using pixel-level fusion combining the richest texture information per wavelength for each textural region. Further descriptions of simultaneous NIR+VW iris acquisition systems can be found in Chou et al. [5] and Gong et al. [7], with the latter giving a good a summary of available multi-spectral iris databases.

Fusion of multispectral information has been the major focus in efforts to capture information from multiple wavelengths: Boyce et al. [1] investigated NIR+VW iris fusion for recognition and cross-spectral applications using a collected multispectral (red at $670 \mathrm{~nm}$, green at $540 \mathrm{~nm}$, blue at $475 \mathrm{~nm}$ and near infrared at $800 \mathrm{~nm}$ ) iris images database with 24 users reporting higher accuracy for the red channel compared to green and blue channels for Daugmans 2DGabor features [6]. ROC curves on cross-spectral matching (matching iris images across different wavelengths) revealed decreasing performance with increasing differences between wavelengths. Fusion tests were limited to scorelevel (sum rule) combination, revealing highest accuracy for the fusion between the NIR, red and green channels. Wavelengths beyond $900 \mathrm{~nm}$, examining 8 bands (100nm) within the $950-1650 \mathrm{~nm}$ spectrum were further investigated by Ross et al. [19]. Facing the challenges of illumination (using a tungsten-krypton light) and segmentation (high variance in contrast between sclera and iris), experiments on a database of 25 users confirmed the possibility of performing crossspectral matching beyond $900 \mathrm{~nm}$ and well separation in 5-wavelength fusion configuration. In Ngo et al. [15] the design and implementation of a multispectral iris images acquisition system is given, employing 8 wavelengths in the $405 \mathrm{~nm}$ to $1070 \mathrm{~nm}$ range. It is claimed that true matches degrade with wavelength difference and experiments illustrate the best accuracy around $800 \mathrm{~nm}$ on the initial provided dataset of 392 images from 6 subjects. Cross-spectral application severely shifted genuine distributions towards (zero-effort) impostors. Ives et al. [10] present their multispectral acquisition system and database of 16 users (1859 images) in the 405-1070nm range. Results confirm a systematic bias if automatic segmentation is used in contrast to hand-segmented data and they report 0.237 vs. 0.193 best average genuine Hamming Distance (HD) comparing $590 \mathrm{~nm}$ with $910 \mathrm{~nm}$. Further cross-spectral genuine HD performance confirms claims on an increase by wavelength difference by previous authors. In Gong et al. [8] the question on the most important multispectral bands in the range of 420 to $940 \mathrm{~nm}$ is addressed using a dataset of 30 subjects, using agglomerative clustering on 2D PCA. Results indicated three wavelength bands were enough to encode the features, however the database was limited to Asian participants only. Zuo et al. [21] predict the NIR image based on the colour image and report slightly enhanced accuracy compared to matching the red channel only. In their work using 1D Log-Gabor features a feed forward neural network and the database in [1] is used. Shamsafar et al. [20] sug- 


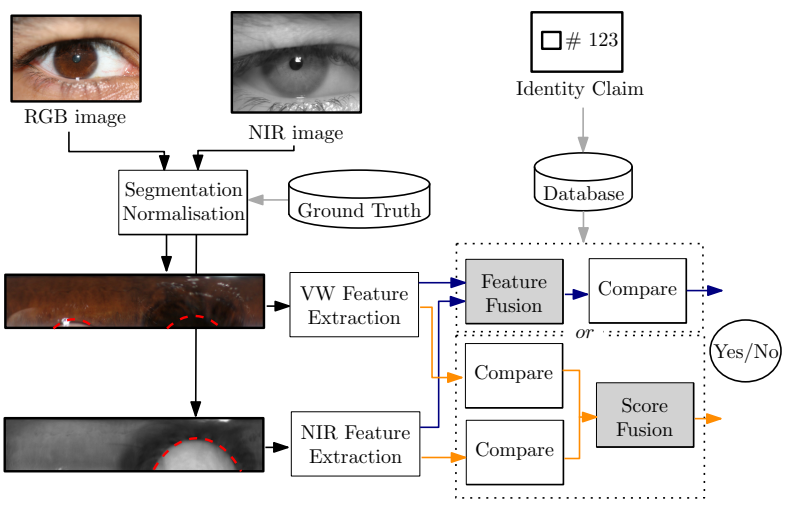

Figure 2: Investigated Multispectral Iris Fusion Setup.

gested a Log-Gabor and Haar-based NIR and VW iris fusion method on the same UTIRIS database used in this paper.

It is worth noticing that employed test databases in the studies above have been limited in the number of tested users and public availability. Hosseini et al. [9] present a collected multispectral database (UTIRIS), consisting of NIR and VW images of 158 eyes from 79 individuals, for which they propose a combination of shape-based features with an overall fusion accuracy of $90 \%$ Genuine Acceptance Rate (1 training, 4 testing images) to 99\% (4 training, 1 testing) at $1 \%$ False Acceptance Rate. In our work we use UTIRIS and obtain higher accuracy, but without the need for any training. Many researchers (as in [15, 10]) highlight, that more research is necessary to obtain further insight in the coherence between irises at different wavelengths.

\section{System Setup}

This paper focuses on the problem of score and featurelevel NIR + VW fusion and cross-spectral performance, following the traditional iris processing chain outlined in [6] with Rubbersheet normalisation of obtained inner pupillary and outer limbic boundaries and generation of a noise mask taking eyelids into account, but with several different feature extraction methods tested in NIR and VW. Fig. 2 illustrates the setup conducting evaluations in verification mode.

For evaluation purposes the open University of Teheran multispectral iris dataset UTIRIS [9] of 1540 images is employed, with VW and NIR images taken from the same individuals in 2 separate sessions. Approximately 5 full-eye images of high quality with $2048 \times 1360$ pixels resolution for VW and 5 images with $1000 \times 776$ pixels resolution for NIR per eye are available for each of the 79 users. VW and NIR images of the same user are paired to form a dataset of 768 templates of 158 classes (removing unpaired samples).

For accurate segmentation and in order not to bias the results (segmentation performance is reported to be strongly affected by the spectral acquisition band [19]), all input images were manually segmented based on an elliptical model.

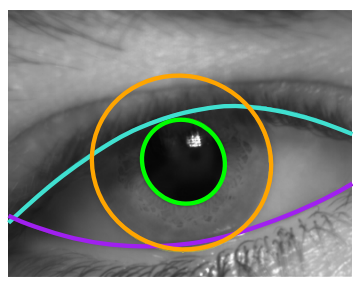

(a) NIR sample

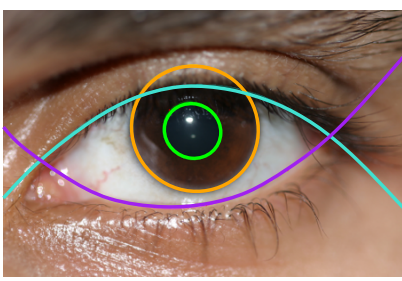

(b) VW sample
Figure 3: Manual UTIRIS segmentation samples.

For this purpose, human segmenters selected a minimum of 5 data points for each of the inner and outer iris boundaries and were shown the (least squares using Fitzgibbion's algorithm) fitted circumference next to the input image. Further, upper and lower eyelids were modelled using 2nd order polynomials (using least-squares fitting) to fit a minimum of 3 labelled datapoints. Fig. 3 illustrates the resulting boundary ellipses and polynomials for two samples. Iris textures are mapped into a normalised polar-unwrapped representation employing Daugman's rubbersheet transform [6] and using a feature-specific target size (see Fig. 2). Contrastlimited adaptive histogram equalisation (CLAHE) is employed to enhance textures.

\subsection{Employed Features}

As feature extractors we employed the publicly available USIT [18] software for LG, QSW and DCT, and our reimplementation of Daugman's [6] approach, 2DG. The latter 2DG feature is the feature of choice in most commercial implementations, using the spatial-frequency information at pixels $(x, y)$ encoded by $2 \mathrm{G}$ Gabor filters:

$$
G(x, y)=e^{-\pi \frac{x^{2}+y^{2}}{\sigma^{2}}} e^{2 \pi f i(x \cos \theta+y \sin \theta)}
$$

By choosing appropriate filter parameters, they have similar behaviour to multi-resolution analysis [11], such as wavelet transform. In the present investigation the 2D Gabor filter parameters were found by performing a direct search in the two-dimensional space defined by the size and frequency parameters, for a fixed orientation of the filter. However, in contrast to QSW and LG, parameters were optimised for VW instead of NIR data to reflect this impact on multispectral performance. The algorithm uses a $160 \times 100$ pixels sized normalised input and produces a 3,000 bits code.

The QSW feature was proposed by Ma et al. [13] and encodes minima and maxima (alternating a binary sequence whenever a new extremal value is found) from two specific subbands of the iris texture. Subbands are obtained from dividing the texture into ten $1 \mathrm{D}$ signals and applying wavelet transform. The algorithm uses a $512 \times 64$ pixels sized normalised input and produces a 10, 240 bits code.

The LG feature is an adapted version of Masek's 1D Log-Gabor convolution of the $512 \times 64$ texture encoding the phase angle with 2 bits, yielding again 10, 240 bits. 
As an alternative, DCT (following Monro et al. [14]) computes the 1D discrete cosine transform on overlapping diamond-shaped image patches. The feature uses the $512 \times$ 64 pixels sized normalised input and produces a $7 \times 3,072$ bits code (for each of 7 pixel shifts).

As comparators, for QSW, LG and 2DG we use the minimum fractional HD (using \pm 4 shifts in either direction) as a measure of (dis-)similarity between feature vectors codeA and codeB, and corresponding code masks (maskA, maskB identifying out-of-iris bits using ground-truth) [6]:

$$
H D=\frac{\|(\operatorname{code} \mathrm{A} \oplus \operatorname{code} \mathrm{B}) \cap \operatorname{maskA} \cap \operatorname{maskB}\|}{\| \text { maskA } \cap \text { maskB } \|} .
$$

For the DCT feature, the comparator defined in [14] is employed.

\subsection{Investigated Fusion Methods}

Comparison in multispectral systems may refer to either gallery or probe samples available in multiple channels, or both. Let $w, x, y, z \in\{N I R, R, G, B\}$ denote channels, when assessing cross-channel performance we investigate scores $s_{x, y}$ for comparing a probe image channel $y$ with gallery image channel $x$, or short $s_{x}:=s_{x, x}$ for intrachannel. Note, that in general even for symmetric matchers $s_{x, y} \neq s_{y, x}$ as different images are involved (but distributions in our experimental setup are similar). In score-level fusion, any subset of the $4^{2}$ scores could be combined, but usually, focus is on combining intra-channel scores: in experiments, we focus on 2-channel fusion combining score vectors $\vec{s}_{x, y}:=\left\{s_{x}, s_{y}\right\}, 3$-channel $\vec{s}_{x, y, z}$ and 4-channel $\vec{s}_{w, x, y, z}$ vectors. As combination technique we employ sum-rule fusion: $C_{\text {sum }}(\vec{s}):=\frac{1}{n} \sum_{i=1}^{n} s[i]$, where $s[i]$ denotes the $i$-th element in the score vector of $n$ elements.

The paper aims to consider feature-level approaches, however, simple concatenation of features in case of employing the fractional HD as feature should (neglecting the presence of bit-shifts to optimise the alignment) yield similar resuls as for score-level fusion. Further, concatenation generally increases the length of the feature vector $\vec{f}$, whereas the approach studied in this work selects bits from $n$ channels $\vec{F}=\left(\vec{f}_{x}, \vec{f}_{y}, \ldots, \vec{f}_{z}\right)$ keeping the length $m$ of the feature vector: $C_{b i t s e l}(\vec{F})[i]:=\vec{F}[\operatorname{sel}(1, n)][i]$ for all $i$ with $1 \leq i \leq m$. This approach follows [17], but applied to multiple spectra and instead of training uses a random selection of bits ( $\mathrm{sel}$ is the random function returning equally distributed natural numbers between 1 and $n$ ), reported to perform remarkably well in incremental iris recognition [18] without any training.

\section{Experimental Results}

Investigating a series of questions related to multispectral fusion performance, we employ Receiver Operating

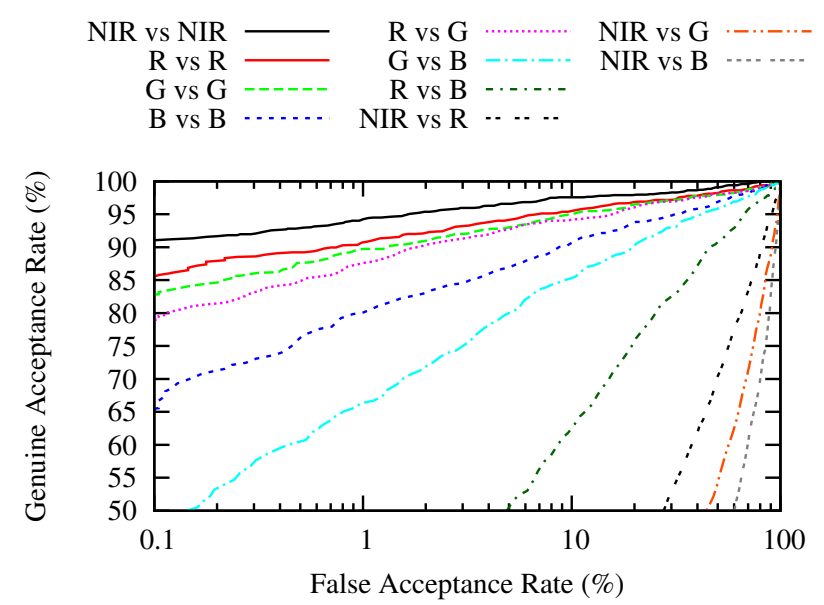

(a) LG

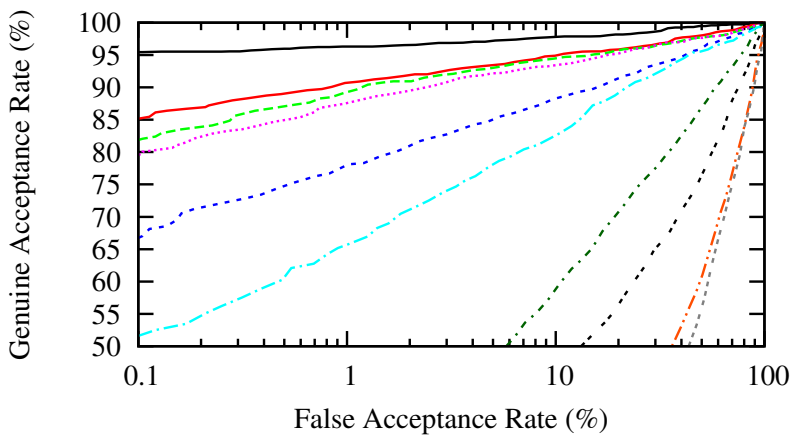

(b) QSW

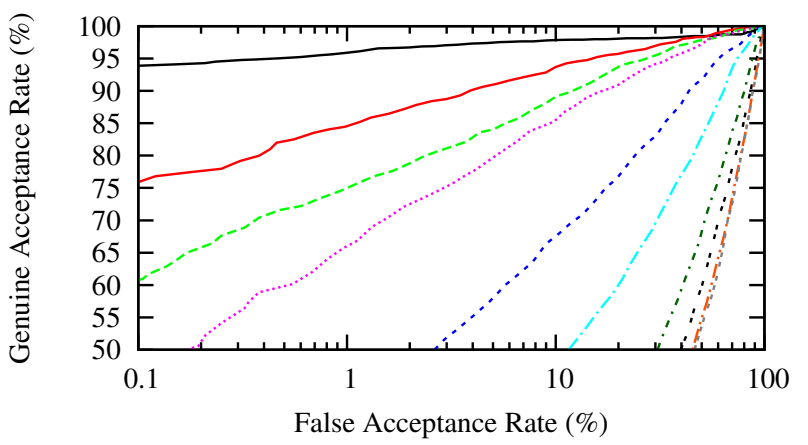

(c) DCT

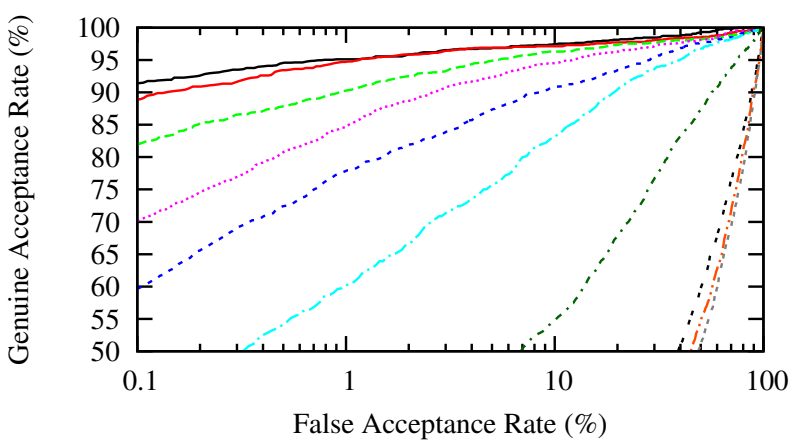

(d) $2 \mathrm{DG}$

Figure 4: Single vs. cross-spectral ROCs for different features (a) LG, (b) QSW, (c) DCT and (d) 2DG on UTIRIS. 
Table 1: Obtained Equal error rates [\%] per feature type when comparing different channels on UTIRIS.

\begin{tabular}{|c|c|c|c|c|c|c|c|c|c|c|c|c|c|c|c|c|}
\hline & \multicolumn{4}{|c|}{ LG } & \multicolumn{4}{|c|}{ QSW } & \multicolumn{4}{|c|}{ DCT } & \multicolumn{4}{|c|}{$2 \mathrm{DG}$} \\
\hline & NIR & Red & Green & Blue & NIR & Red & Green & Blue & NIR & Red & Green & Blue & NIR & Red & Green & Blue \\
\hline NIR & 3.76 & 39.05 & 47.79 & 55.15 & 3.14 & 33.26 & 43.84 & 46.97 & 2.95 & 44.97 & 47.57 & 48.12 & 3.29 & 44.85 & 47.60 & 49.43 \\
\hline $\operatorname{Red}$ & & 5.56 & 6.38 & 22.28 & & 6.16 & 7.14 & 25.11 & & 7.63 & 12.32 & 40.36 & & 3.42 & 6.56 & 26.55 \\
\hline Green & & & 6.36 & 12.76 & & & 6.36 & 13.98 & & & 10.62 & 30.26 & & & 5.08 & 13.56 \\
\hline Blue & & & & 9.63 & & & & 11.24 & & & & 21.73 & & & & 9.40 \\
\hline
\end{tabular}

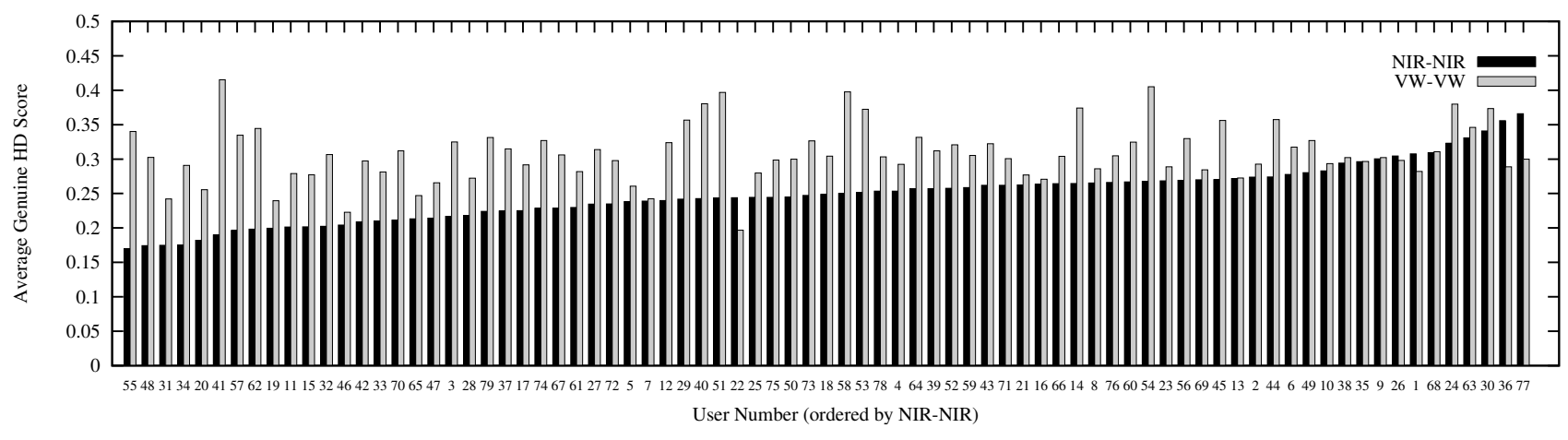

Figure 5: LG on UTIRIS: NIR goats (high average genuine HD) generally do not automatically become VW (red) goats.

Characteristics (ROC) illustrating pairs of Genuine Acceptance Rate (GAR, the rate of genuine users being accepted) and (zero-effort) False Acceptance Rate (FAR, rate of impostors being accepted). As main performance indicator we refer to Equal Error Rate (EER, the rate where 1-GAR equals FAR).

\subsection{Intra versus Cross-spectral Performance}

In a first experiment we focused on the question: How is intra and inter-channel performance affected for different types of features? ROCs in Fig. 4 illustrate all tested intrachannel and cross-channel applications per feature type on the UTIRIS database and Table 1 summarises EERs for a better overview, with entries along the diagonal being intrachannel comparisons (best results in bold).

At first, we see that all algorithms, LG, QSW, DCT and 2DG perform in a rather narrow band for the NIR channel (2.95-3.76\% EER), however there are clear differences for other channels: the VW-optimised 2DG implementation revealed the best intra VW-channel performance for all channels (3.42\% Red, 5.08\% Green, 9.40\% Blue EERs), which suggests training on VW data is beneficial, as it had no negative impact on NIR performance (compare 2DG with LG). The long-bitcode implementations LG and QSW performed quite comparable for intra-channel (5.56-6.16\% Red, 6.36\% Green and 9.63-11.24\% Blue EERs). DCT performed significantly worse, especially for the Blue channel $(21.73 \%$ EER). As a reason for this behaviour the higher variation in gradient orientation in VW channels (as identified in [16]) impacting on features could be argued.
Cross-spectral performance is very low across the board and especially between NIR and RGB channels (33.26$55.15 \%$ EER), certainly also attributable to the pairing of images in non-synchronous acquisition. However, this underlines the needs for efficient transformation mappings before feature extraction in cross-spectral application and suggests that complementary information is present in different channels. Results support the assumption of increasing error rates with increasing difference in channel wavelength. However, we found that the difference between Red and Green was much less pronounced (factors 1.1-1.6 between cross-spectral Red vs. Green and intra-spectral Red and Green only performance, depending on feature) than between Green and Blue (observed factors 1.7-2.1), despite comparable difference in wavelength (typical peak sensitivity Blue at $460 \mathrm{~nm}$, Green at $540 \mathrm{~nm}$, and Red at $620 \mathrm{~nm}$ ).

\subsection{On Doddington's Goats in Multiple Channels}

The inferior cross-spectral performance and presumably augmenting information in the VW and NIR channels as illustrated in Fig. 1 suggests to investigate the question: Are difficult subjects in one spectrum also difficult in another? We analysed average genuine HD for individual users and investigated the corresponding user rank with regards to this metric (see Fig. 5 plotting LG scores in both spectra). Results indicated, however, that a low average genuine HD score for NIR does not necessarily indicate a high or low score in VW (Pearson correlation coeff. 0.19). Similar behaviour is observed for the DCT and Gabor-based features. 
Table 2: Score-level Fusion performance on UTIRIS.

\begin{tabular}{rcccc}
\multicolumn{5}{c}{ Equal-error rate [\%] } \\
Combined Channels & LG & QSW & DCT & 2DG \\
\hline NIR + R & 0.93 & 0.60 & $\mathbf{0 . 8 2}$ & $\mathbf{0 . 7 4}$ \\
NIR + G & 0.87 & 0.67 & 1.33 & 0.94 \\
NIR + B & 1.27 & 1.26 & 2.52 & 1.35 \\
R + G & 5.64 & 5.61 & 8.04 & 3.49 \\
R + B & 5.34 & 5.96 & 9.29 & 4.21 \\
G + B & 6.66 & 6.71 & 12.51 & 6.11 \\
\hline R + G + B & 5.49 & 5.78 & 9.23 & 4.29 \\
NIR + R + G & 0.88 & 0.64 & 1.20 & 1.56 \\
NIR + R + B & $\mathbf{0 . 7 0}$ & $\mathbf{0 . 5 9}$ & 1.46 & 1.34 \\
NIR + G + B & 0.79 & 0.73 & 1.75 & 1.61 \\
\hline NIR + R + G + B & 1.04 & 0.63 & 1.76 & 1.95 \\
\hline & & & &
\end{tabular}

\subsection{Multispectral Score-level Fusion}

We further investigate the question: Which features and combinations thereof are most discriminatory for different wavelengths? A test of all possible channel combinations (see Table 2, best results in bold) for each of the features LG, QSW, DCT and 2DG revealed the following results: Among all tested 2-channel combinations, the best overall performance was provided by the combination of the NIR and Red channels (LG provided slightly better results for NIR and Green with $0.87 \%$ vs. $0.93 \%$ EER) with EERs in the range $0.6-0.93 \%$, corresponding to a significant improvement (factors of 3.6-4.4) compared to single-channel NIR performance. Improvement was most imminent for the QSW feature, followed by 2DG, LG and DCT, but despite their different nature (especially DCT), revealed a similar level of improvement. Combinations of the color channels were not as successful and mostly did not improve the result of single channels (DCT and 2DG), only marginal improvement in case of $\mathrm{R}+\mathrm{G}(\mathrm{QSW})$ and $\mathrm{R}+\mathrm{B}(\mathrm{QSW}$ and $\mathrm{LG})$ could be observed. Consequently, 3-channel fusion delivered better results for only these two features, LG and QSW $(0.7 \%$ EER for LG and $0.59 \%$ EER for QSW, both when combining NIR+R+B). Especially the lower performance of $2 \mathrm{DG}$ for 3-channel fusion (1.34\% EER for NIR+R+B) is surprising, given that this algorithm was tuned for VW and showed better performance on individual color channels. Best 2channel and 3-channel performance ROCs on UTIRIS are illustrated in Fig. 6. While possibilities in score-level combination are limited compared to feature-level fusion, in this configuration it is advisable to aim for NIR+Red channel fusion taking the additional processing and storage overhead for additional channels into account.

\subsection{Multispectral Selective Bits Fusion}

Finally, we evaluated the approach of multispectral selective bits fusion on the LG and QSW features. As the

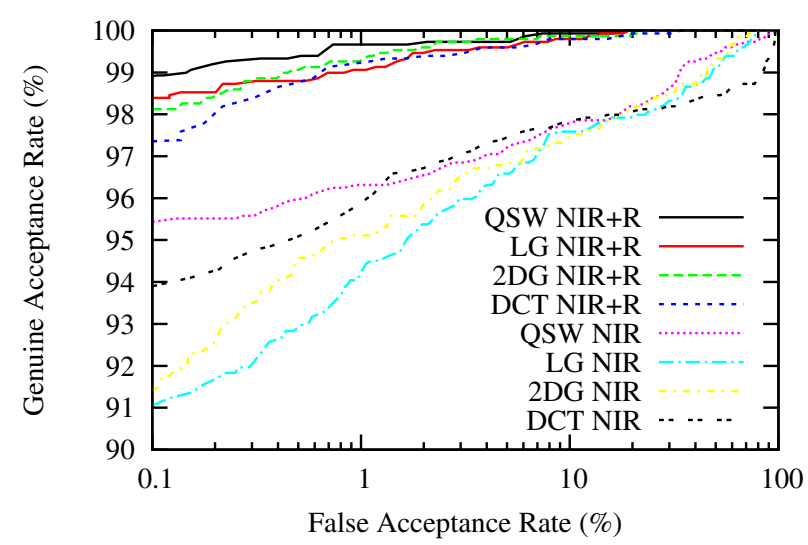

(a) Best 2-channel (NIR+R) UTIRIS performance

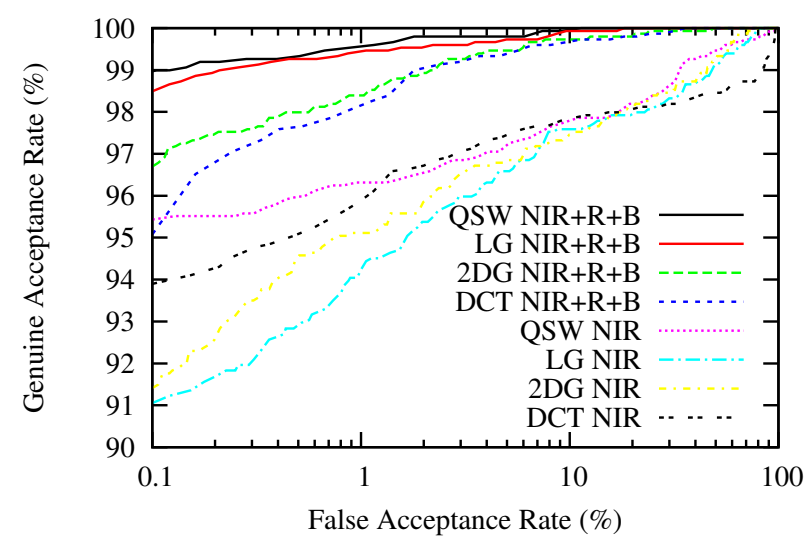

(b) Best 3-channel (NIR+R+B) UTIRIS performance

Figure 6: ROC for score-level multispectral fusion.

combination of NIR and Red turned out to be the most effective combination of channels we also selected bits randomly from these two channels. In both cases, results outperformed single-channel codes (2.25\% vs. $3.14 \%$ EER for QSW, $1.52 \%$ vs. 3.76\% EER for LG) without increasing overall length. A caveat in the bits fusion process is the ability to keep rotation invariance. The possibility of alignment using shifts would be lost in case of bitwise merger and can be avoided in merging batchwise (we used 8 bit), which keeps up the possibility to a limited number of shifts. Especially for cross-spectral application, multispectral selective bits fusion delivers much better results than unmerged features, with EERs of $8.89 \%$ NIR+R vs. R and $5.16 \%$ NIR+R vs. NIR (compare with $33.26 \%$ NIR vs. R) for LG and 9.8\% NIR+R vs. $\mathrm{R}$ and $4.55 \% \mathrm{NIR}+\mathrm{R}$ vs. NIR (compare with 39.05\% NIR vs. R) for QSW. Figure 7 illustrates corresponding ROCs. Partial matching of concatenated codes without reordering (first 50\% $\mathrm{R}+$ last 50\% NIR) resulted in $3.13 \%$ EER for QSW and 2.08\% EER for LG. 


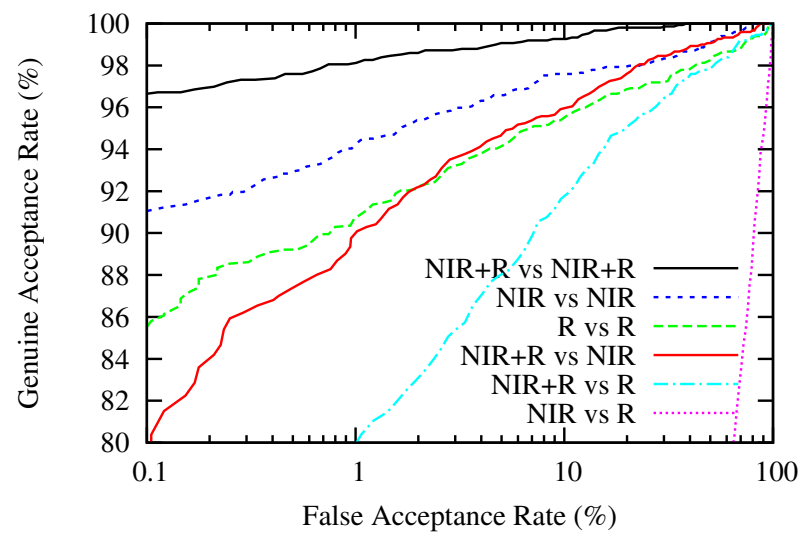

Figure 7: ROC for feature-level multispectral fusion on LG.

\section{Conclusion and Future Work}

In this work we studied the impact of score-level fusion and cross-spectral performance in multispectral iris recognition systems, investigating Log Gabor (LG), Waveletbased (QSW), Discrete Cosine Transform (DCT) and 2D Gabor (2DG) feature types. Results on the public UTIRIS multispectral database indicated, that features can be rather susceptible to spectral channels (such as DCT delivering best NIR but worst RGB performance). Cross-spectral performance turned out to be highly challenging (EERs $>33 \%$ for comparing NIR against RGB channels across feature types), confirming increases in EERs as the difference in wavelength increases, but further also indicating a more pronounced degradation for Green versus Blue compared with Red versus Green intra-color cross-spectral application. Score-level fusion delivered best results for NIR+R+B for 3-channel $(0.59 \%$ EER, 99\% GAR at $0.1 \%$ FAR with QSW), closely followed by NIR+R in 2-channel configuration with the latter providing most stable rates across all feature types (0.6 - 0.93\% EER, $97-99 \%$ GAR at $0.1 \%$ FAR). Sensitivity to channel improvement was found to be severely affected by the feature type. The suggested multispectral random selective bits fusion technique was able to improve accuracy (1.52\% vs. $3.76 \%$ EER, $97 \%$ vs. $91 \%$ GAR at $0.1 \%$ FAR for LG) with the benefit of unmodified feature length. In the future, we plan to investigate learningbased methods to select bits from multiple channels.

\section{Acknowledgements}

This work was supported by the EU FASTPASS project under grant agreement 312583 .

\section{References}

[1] C. Boyce, A. Ross, M. Monaco, L. Hornak, and X. Li. Multispectral iris analysis: A preliminary study. In Proc. Conf. on Comp. Vision and Pattern Rec. WS, pages 51-51, 2006.
[2] M. Burge and M. Monaco. Multispectral iris fusion and cross-spectrum matching. In M. Burge and K. Bowyer, editors, Handbook of Iris Recognition. Springer, 2013.

[3] M. J. Burge and M. K. Monaco. US Pat. 0279790. Multispectral Iris Fusion for Enhancement and Interoperability, 2009.

[4] R. Chen, X. Lin, and T. Ding. Liveness detection for iris recognition using multispectral images. Pattern Recognition Letters, 33(12):1513 - 1519, 2012.

[5] C.-T. Chou, S.-W. Shih, W.-S. Chen, V. Cheng, and D.-Y. Chen. Non-orthogonal view iris recognition system. IEEE Trans. Circ. Syst. Video Techn., 20(3):417-430, March 2010.

[6] J. Daugman. How iris recognition works. IEEE Trans. on Circiuts and Systems for Video Technology, 14(1), 2004.

[7] Y. Gong, D. Zhang, P. Shi, and J. Yan. High-speed multispectral iris capture system design. IEEE Trans. on Instrumentation and Measurement, 61(7):1966-1978, 2012.

[8] Y. Gong, D. Zhang, P. Shi, and J. Yan. Optimal wavelength band clustering for multispectral iris recognition. Appl. Opt., 51(19):4275-4284, 2012.

[9] M. Hosseini, B. Araabi, and H. Soltanian-Zadeh. Pigment melanin: Pattern for iris recognition. IEEE Trans. on Instrumentation and Measurement, 59(4):792 -804, 2010.

[10] R. Ives, H. Ngo, S. Winchell, and J. Matey. Preliminary evaluation of multispectral iris imagery. In Proc. IET Conf. on Image Processing, pages 1-5, July 2012.

[11] V. Kyrki, J.-K. Kamarainen, and H. Kälviäinen. Simple gabor feature space for invariant object recognition. Pattern Recognition Letters, 25(3):311 - 318, 2004.

[12] G. B. Lemos, V. Borish, G. D. Cole, S. Ramelow, R. Lapkiewicz, and A. Zeilinger. Quantum imaging with undetected photons. Nature, 512:409-412, 2014.

[13] L. Ma, T. Tan, Y. Wang, and D. Zhang. Efficient iris recognition by characterizing key local variations. IEEE Transactions on Image Processing, 13(6), 2004.

[14] D. M. Monro, S. Rakshit, and D. Zhang. Dct-based iris recognition. IEEE Trans. Patt. Anal. Mach. Int., 29(4), 2007.

[15] H. Ngo, R. Ives, J. Matey, J. Dormo, M. Rhoads, and D. Choi. Design and implementation of a multispectral iris capture system. In Proc. of CSSP, pages 380-384, Nov 2009.

[16] P. Radu, K. Sirlantzis, G. Howells, S. Hoque, and F. Deravi. Information fusion for unconstrained iris recognition. Int'l $J$. of Hybrid Inf. Techn., (4), 2011.

[17] C. Rathgeb, A. Uhl, and P. Wild. On combining selective best bits of iris-codes. In Proc. Biometrics and ID Management WS, pages 227-237. 2011.

[18] C. Rathgeb, A. Uhl, and P. Wild. Iris Recognition: From Segmentation to Template Security, volume 59 of Advances in Information Security. Springer, 2012.

[19] A. Ross, R. Pasula, and L. Hornak. Exploring multispectral iris recognition beyond 900nm. In Proc. Int'l Conf on Biometrics: Th., App., and Syst., pages 1-8, Sept 2009.

[20] F. Shamsafar, H. Seyedarabi, and A. Aghagolzadeh. Fusing the information in visible light and near-infrared images for iris recognition. Mach. Vis. App., 25(4):881-899, 2014.

[21] J. Zuo, F. Nicolo, and N. Schmid. Cross spectral iris matching based on predictive image mapping. In Proc. Int'l Conf. on Biometrics: Th., App., and Syst., pages 1-5, Sept 2010. 